\title{
Encouraging Early Outcomes with Image Guided Pencil Beam Proton Therapy for Cranio-Spinal Irradiation and Unique Considerations for Paediatric Population - First Report From India
}

Utpal Gaikwad

Apollo Proton Cancer Centre

Noufal MP

Apollo Proton Cancer Centre

Jacinthlyn Sylvia

Apollo Proton Cancer Centre

Ashok K Reddy

Apollo Proton Cancer Centre

Pankaj Kumar Panda

Apollo Proton Cancer Centre

Srinivas Chilukuri

Apollo Proton Cancer Centre

Dayananda Sharma

Apollo Proton Cancer Centre

Rakesh Jalali ( $\square$ rjalali@apollohospitals.com )

Apollo Proton Cancer Centre

Research Article

Keywords: Proton, CSI, Pediatric, Medulloblastoma, Ependymoma

Posted Date: February 23rd, 2022

DOI: https://doi.org/10.21203/rs.3.rs-1375776/v1

License: @ (i) This work is licensed under a Creative Commons Attribution 4.0 International License.

Read Full License 


\section{Abstract \\ Background}

To report our experience with image guided pencil beam proton beam therapy (PBT) for craniospinal irradiation (CSI).

\section{Materials and Methods}

Between January 2019 to Dec 2021, we carried out a detailed audit of the first forty patients treated with PBT. We had recorded acute toxicities, reporting early outcomes and discuss limitations of current contouring guidelines during CSI PBT planning.

\section{Results}

Median age of the patient cohort was 8 years, and histologies include 20 medulloblastoma, 7 recurrent ependymoma, 3 pineoblastoma, 3 were germ cell tumors and remaining 7 constituted other diagnoses. Forty percent patients received concurrent chemotherapy. Median CSI dose was 23.4 GyE (Gray Equivalent; range 21.6-35). Thirty-five patients (87.5\%) completed their CSI without interruption, 5 required hospital admission. No patient had grade $2 />$ weight loss during the treatment. Forty-five percent (18) developed grade 1 haematological toxicities and 20\% (8) developed grade 2 or 3 toxicities; none had grade 4 toxicities. At median follow up of 12 months, $90 \%$ patients are alive of whom $88.9 \%$ are having local control. Special consideration with modification in standard contouring used at our institute helped in limiting acute toxicities in paediatric CSI patients.

\section{Conclusion}

Our preliminary experience with modern contemporary PBT using pencil beam technology and daily image guidance in a range of tumours suitable for CSI is encouraging. Patients tolerated the treatment well with acceptable acute toxicity and expected short-term survival outcome. In paediatric CSI patients, modification in standard contouring guidelines required to achieve better results with PBT.

\section{Introduction}

Craniospinal irradiation (CSI) is an integral component in the standard of care management in a range of CNS tumours including medulloblastoma, pineoblastoma, selected ependymomas, intracranial germ cell tumours, supra-tentorial embryonal tumours etc $(1-4)$. Conventional photon based CSI has been shown to be associated with fair amount of acute toxicities due to large volume of treatment and significant amount of vertebral bone marrow irradiation, causing treatment gaps, risk of infections, hospital admission during treatment particularly with concurrent chemotherapy. (5) Over last six decades there 
have been evolution of changes in CSI planning and implementation and current availability of modern proton therapy is emerging as the standard of care whenever available. $(6,7)$ Proton therapy, by virtue of its physical properties provides dosimetric over modern photon therapy techniques, which are well established.(8) Mahajan et al in review of CSI using proton therapy reported that, proton therapy was associated with lower acute toxicities and emphasised that further refinement of proton therapy with the use of pencil beam scanning technique might allow further reduction in acute toxicities. In addition, the incorporation of on-board volumetric imaging techniques such as cone beam CT, CT on rails and surface imaging, facilitates daily accurate patient positioning.

We hereby report our institutional experience of CSI using image guided, pencil beam scanning proton therapy in first forty patients, their acute toxicities and early outcomes.

\section{Materials And Methods}

Forty patients with various histologies, who underwent CSI using proton therapy after detailed discussion in the institutional multidisciplinary tumour and proton boards are included in this study. All patients after pre planning audit amongst treating physician, planning physicist and radiation therapist, underwent radiotherapy simulation. A customized neck rest (Moldcare Cushion Qfix, Avondale, USA) and thermoplastic mask (Fiberplast, QFix, Avondale, USA) were used for immobilization of head neck region, with arms by side, in supine position. A customized Vacloc either full body or till pelvis, was made for lower body immobilization. Computed Tomography (CT) simulation was performed with $3 \mathrm{~mm}$ slice thickness axial images using Canon Aquilion LB CT scanner (Canon Medical Systems, Singapore). Contrast enhanced MRI (Magnetic Resonance Imaging) scan was acquired in treatment position for tumour bed delineation with the aid of pre op MRI scan and planning MRI sequences. Craniospinal target delineation was done as per standard International Society of Paediatric Oncology (SIOP) guidelines. (9) In adult patients, clinical target volume for brain (CTV Brain) delineated which was covering whole brain and dural cuffs of cranial nerves, while CTV spine was covering entire subarachnoid space and nerve roots. Planning target volume (PTV) was generated by expanding CTV brain by $3 \mathrm{~mm}$ and CTV spine by 5 $\mathrm{mm}$. For paediatric patients, standard contouring guidelines (SIOP and COG) recommends delineation of whole brain with dural extensions as CTV brain, whole vertebral body as spinal CTV. Brain CTV with $3 \mathrm{~mm}$ circumferential margin and spinal CTV with $3 \mathrm{~mm}$ circumferential margin should be combined to generate CSI PTV (9). After a detailed discussion among treating physicians, planning physicists and radiotherapy technologists, we modified our approach for paediatric patients; delineated the whole spinal canal with nerve extensions and generated a robust CSI plan with acceptable coverage of whole vertebral body (Fig. 2; IIIA, IIIB). This approach was mainly adapted to reduce anterior OAR dose (oesophagus, midline mucosa, thyroid, bowel bag etc) without significant underdosing or sharp dose gradient in vertebral body region which is known to cause long term skeletal abnormalities. All OARs were drawn and standard dose constraints were prescribed and handed over to physicist to generate CSI plans followed by sequential tumour bed boost plan. 
Robustly optimized hybrid proton plans were generated for all patients as per institutional planning protocol using RayStation (Version 9.0, RaySearch Laboratories AB, Stockholm, Sweden) treatment planning system (TPS) (10). Two posterior oblique fields for brain and one or two posterior beams for spine were used depending on length of the spine. All plans were evaluated for target coverage and OAR dose using standard dose volume indices with robustness of $3 \mathrm{~mm}$ translational errors and $3.5 \%$ range uncertainties (11).

After radiation plan finalization, and patient specific quality assurance (PSQA) of finalized plan as per institutional protocol, all patients received CSI and sequential tumour bed boost. Daily plan implementation was done after patient position verification using on board CBCT and kilovoltage (KV) $\mathrm{x}$ rays under supervision of treating physician and planning physicist.

Patients underwent quality assurance $\mathrm{CT}$ scans at regular interval either after 5-7 fractions or according to $\mathrm{CBCT}$ / clinical findings. All patients were reviewed at least once weekly during radiation therapy, 4 weeks after completion of treatment and thereafter at 3 monthly intervals. Acute adverse events were documented according to Common Terminology Criteria for adverse Events (CTCAE v5.0).

\section{Results}

Of the 40 patients treated with CSI using proton beam therapy at our institute from February 2019 to December 2021, 33 (83.3\%) were age less than 18 years at the time of treatment. Their demographic data, details of diagnosis, molecular subgroups, treatment received are summarized in Table 1 and Fig. 1. Dosimetric data for these patients is summarized in Table 2. 
Table 1

- Demographic and treatment details

\begin{tabular}{|c|c|}
\hline Median Age & 8 years ( 1.5 to 37 years) \\
\hline \multirow[t]{3}{*}{ Age distribution } & $0-6$ years $-15(37.5 \%)$ \\
\hline & $7-14$ years $-16(40 \%)$ \\
\hline & $>14$ years $-9(22.5 \%)$ \\
\hline Sex Ratio (M:F ) & $32: 8$ \\
\hline \multirow[t]{6}{*}{ Histopathology } & $20(50.0 \%)$ - Medulloblastoma \\
\hline & 7 (17.5\%) - Recurrent Ependymoma \\
\hline & 3 (7.5\%) - Pineoblastoma \\
\hline & $3(7.5 \%)$ - Germ cell tumors \\
\hline & $7(17.5 \%)$ - Others \\
\hline & (ATRT, PNET, APL with CNS involvement and neuroblastoma) \\
\hline \multirow[t]{2}{*}{ Reirradiation } & Yes $-7(17.5 \%)$ \\
\hline & No - $33(82.5 \%)$ \\
\hline \multirow[t]{2}{*}{ Anesthesia required } & $6(15 \%)$ - For simulation and complete treatment \\
\hline & $2(5 \%)$ - For simulation only \\
\hline \multirow[t]{4}{*}{ Radiation dose delivered } & Median dose 23.4 GyE \\
\hline & $23.4 \mathrm{GyE}-20(50 \%)$ \\
\hline & 35 GyE - 10 (25\%) \\
\hline & Other - $10(25 \%)$ \\
\hline \multirow[t]{2}{*}{ Concurrent chemotherapy } & Yes - $16(40 \%)$ \\
\hline & No $-24(60 \%)$ \\
\hline \multirow[t]{2}{*}{ Chemotherapy agents used } & Vincristine (81.2\%) \\
\hline & Carboplatin (18.8\%) \\
\hline
\end{tabular}


Table 2

- Radiation dose details and acute toxicities

\begin{tabular}{|c|c|c|c|}
\hline & CSI (Total) & $\begin{array}{l}\text { CSI } \\
\text { (>14 years) }\end{array}$ & $\begin{array}{l}\text { CSI } \\
\text { (<14 years) }\end{array}$ \\
\hline$n$ & 40 (100\%) & $9(22.5 \%)$ & 31 (77.5\%) \\
\hline PTV CSI D95 (Avg) & $97.4 \%$ & $99.2 \%$ & $96.6 \%$ \\
\hline CTV CSI D98 (Avg) & $98.6 \%$ & $99.1 \%$ & $97.2 \%$ \\
\hline Lens (D max) Avg & 2.96 GyE (1.76\%) & 2.96 GyE (1.76\%) & 2.96 GyE (1.76\%) \\
\hline Cornea (D Mean) Avg & 1.68 GyE (1.34\%) & 1.68 GyE (1.34\%) & 1.68 GyE (1.34\%) \\
\hline Cochlea (D Max) Avg & 27.3 GyE (93.5\%) & 27.3 GyE (93.5\%) & 27.3 GyE (93.5\%) \\
\hline Parotids (D mean) Avg & $\begin{array}{l}10.67 \mathrm{GyE} \\
(4.63 \%)\end{array}$ & 0.44 GyE (0.01\%) & $12.6(37 \%)$ \\
\hline Esophagus (D Max) Avg & $\begin{array}{l}20.31 \mathrm{GyE} \\
(61.6 \%)\end{array}$ & $0.00(0)$ & 27.7 GyE (84\%) \\
\hline Lung (D mean) Avg & 0.65 GyE (0.01\%) & 1.85 GyE $(0.07 \%)$ & 3.22 GyE (0.09\%) \\
\hline Kidney (D mean) Avg & 1.85 GyE (0.08\%) & 2.05 GyE $(0.09 \%)$ & 7.78 GyE (0.23\%) \\
\hline Heart (D mean) Avg & 0 & 0 & 0 \\
\hline Bowel Bag (D mean) Avg & $\begin{array}{l}0.18 \mathrm{GyE} \\
(0.007 \%)\end{array}$ & 0.01 GyE (0) & $0.21 \mathrm{GyE}(0.006 \%)$ \\
\hline Liver (D mean) Avg & 0 & 0 & 0 \\
\hline Gonads (D mean) Avg & 0 & 0 & 0 \\
\hline Dermatitis & $20(50 \%)$ & $4(44.4 \%)$ & $17(54.8 \%)$ \\
\hline$\nabla$ Grade 1 & $20(50 \%)$ & $5(56.6 \%)$ & $14(45.2 \%)$ \\
\hline$\nabla$ Grade 2 & 0 & 0 & 0 \\
\hline \multicolumn{4}{|l|}{$\otimes$ Grade 3} \\
\hline Mucositis & $8(20 \%)$ & $1(11.1 \%)$ & $7(22.5 \%)$ \\
\hline$\nabla$ Grade 1 & $2(5 \%)$ & $1(11.1 \%)$ & $2(6.5 \%)$ \\
\hline$\otimes$ Grade 2 & $1(2.5 \%)$ & 0 & $1(3.2 \%)$ \\
\hline$\nabla$ Grade 3 & & & \\
\hline
\end{tabular}




\begin{tabular}{|c|c|c|c|}
\hline & CSI (Total) & $\begin{array}{l}\text { CSI } \\
(>14 \text { years })\end{array}$ & $\begin{array}{l}\text { CSI } \\
\text { ( }<14 \text { years) }\end{array}$ \\
\hline Weight loss & $3(7.5 \%)$ & 0 & $3(9.6 \%)$ \\
\hline$\nabla$ Grade 1 & 0 & 0 & 0 \\
\hline \multicolumn{4}{|l|}{$\nabla$ Grade 2} \\
\hline Nausea & $6(15 \%)$ & 0 & $6(19.4 \%)$ \\
\hline$\nabla$ Grade 1 & 0 & 0 & 0 \\
\hline \multicolumn{4}{|l|}{ Grade 2} \\
\hline Anorexia & $7(17.5 \%)$ & $1(11.1 \%)$ & $6(19.4 \%)$ \\
\hline$\nabla$ Grade 1 & 0 & 0 & 0 \\
\hline \multicolumn{4}{|l|}{$\nabla$ Grade 2} \\
\hline \multirow{2}{*}{$\begin{array}{l}\text { Hospital admissions during } \\
\text { treatment }\end{array}$} & $5(12.5 \%)$ & Nil & $5(16.1 \%)$ \\
\hline & \multicolumn{3}{|c|}{ Average hospital stay $=2.4$ days } \\
\hline \multirow[t]{2}{*}{ Treatment gap during treatment } & $3(7.5 \%)$ & Nil & $3(9.6 \%)$ \\
\hline & \multicolumn{3}{|c|}{ Average treatment gap $=2$ days } \\
\hline
\end{tabular}

Twenty patients (50\%) were diagnosed with medulloblastoma, 7 (17.5\%) had recurrent ependymoma, 3 (7.5\%) pineoblastoma, 3 (7.5\%) were germ cell tumors and remaining 7 (17.5\%) constituted other diagnoses; namely atypical teratoid rhabdoid tumour (ATRT), primitive neuroectodermal tumour (PNET), acute pro-myelocytic leukemia (APL) with CNS involvement, high grade neuroepithelial tumour and neuroblastoma (Fig. 1a). Among medulloblastoma group, majority were group 4 (55\%) followed by SHH (20\%) while WNT and unclassified constituted $10 \%$ each and one patient was with group 3 (Fig. 1b).

Of all patients, $8(20 \%)$ required short general anaesthesia (sGA) during radiation simulation and of these 8 patients 6 required anaesthesia during entire radiation treatment. Two patients after simulation, and few fractions of radiotherapy under SGA, were managed to complete radiation without it. Median CSI dose prescribed was $23.4 \mathrm{GyE}, 40 \%$ received $23.4 \mathrm{GyE}$ and $30 \%$ received $35 \mathrm{GyE}$ at $1.67 \mathrm{GyE}$ per fraction. Sixteen patients $(40 \%)$ received concurrent chemotherapy, of whom 13 received injection vincristine and 3 received injection carboplatin. Of 31 paediatric patients with age less than 14 years, 15 (48.4\%) received concurrent chemotherapy and patients with age more than 14 years, $1(11.1 \%)$ received concurrent chemotherapy. Seven recurrent ependymoma patients received prior focal radiotherapy and median gap between previous radiation and CSI was 3 years (Range $1-5$ years). 
All patients tolerated treatment well, and only five (12.5\%) patients required hospital admission during treatment, all were age less than 14 years. Acute toxicities were documented as per CTCAE v5.0. Only one patient developed grade 3 mucositis during treatment, who was 6 years old boy and was the first patient receiving whole vertebral body CSI. For this patient 3 days of treatment gap and adaptive planning with contour modification was done, after which he completed treatment well. Other than this, grade 1 mucositis was seen in 8 patients and grade 2 mucositis in 2 patients, all were managed conservatively without any treatment gaps. Twenty (50\%) patients developed grade 2 dermatitis while remaining had grade 1 dermatitis, no one had grade 3 or more dermatitis.

No patient in this cohort developed grade 2 or more weight loss during treatment and median weight loss was $4.8 \%$ of baseline weight, 3 patients lost weight more than $10 \%$ of their baseline, all were paediatric age group receiving concurrent chemotherapy. No patient developed grade 2 or more anorexia, nausea vomiting during treatment, grade 1 anorexia was noted in $8(20 \%)$ patients while $6(15 \%)$ had grade 1 nausea vomiting.

Sixty percent (24) developed grade 1 haematological toxicities, of which 6 had grade 1 decrease in haemoglobin, 6 had grade 1 thrombocytopenia and 12 had grade 1 neutropenia. Three patients developed grade 2 neutropenia and 4 patients developed grade 3 neutropenia. No patient developed grade 2 or 3 thrombocytopenia. Six patients had grade 1 haemoglobin decrease, while only patient had grade 2 haemoglobin decrease. All patients who developed grade 3 neutropenia were age less than 14 years and either received prior radiation or concurrent chemotherapy or both. Three of these 4 patients required hospital admission during the treatment, average hospital stay was 2 days and none of them had prolongation of overall treatment. One patient required hospital admission more than once due to VP (ventriculo-peritoneal) shunt blockade.

At median follow up of 10 months, $83.3 \%$ patients are alive and of them $92 \%$ were alive with no evidence of disease (Fig. 3A). We did subgroup analysis, divided patients according to diagnosis in three groups, medulloblastoma, recurrent ependymoma and others and did survival analysis. In medulloblastoma group overall survival was 95\%, in recurrent ependymoma group $71.4 \%$ while in others it was $76.9 \%$ (Fig. 3B).

\section{Discussion}

Benefits of proton therapy across various subsites are well established and its role in low income countries like India is well justifiable (12). As a first modern proton therapy centre in South East Asia, we treated more than 500 patients over past two years and CNS tumours constituted maximum bulk of it. We have reported our preliminary experience in children and young adults $(13,14)$.

CSI involves radiation delivery to entire neuraxis, which includes whole brain with meningeal reflections, entire spinal canal and thecal sac. Most common indication of Cranio-spinal irradiation (CSI) is medulloblastoma followed by tumors involving risk of neuraxial spread such as pineoblastoma, GCT, intracranial PNET (15). Recently in patients with recurrent ependymoma, re-irradiation with CSI and focal 
boost shown benefit in local control and hence preferred treatment (2). Most of these patients are paediatric age group followed by young adults. In paediatric patients as per current standard recommendations (SIOP and COG) target volume should include whole vertebra so as to avoid or minimize risk of radiation induced skeletal growth abnormalities (9).

Delivering radiation to such large volume target is complex procedure and teletherapy machine limitations for such long treatment, warrants use of multiple fields with field junctions. Field junctions, location of vital OARs (Heart, Lungs, Midline mucosa and bowel bag) in close proximity to target and longer survivorship of patients receiving CSI mandates use of highly precise radiation therapy technique. Over last six decades, CSI techniques evolved tremendously and with availability of proton therapy, it is current standard of care whenever available. Proton beam therapy with pencil beam scanning technique has significantly better dose distribution and OAR sparing when compared with modern intensity modulated radiotherapy and passive scattering proton beam therapy.

In this work, we are analysing acute toxicities of CSI using PBT in both young adult and paediatric patients and their comparison with existing results. Like discussed in various dosimetric studies, proton therapy with no exit dose limits radiation to anteriorly placed OARs due to absence of exit dose $(7,16)$. Dose distribution in sagittal and axial sections with OAR sparing achieved for adult patients, paediatric patients with standard approach and our novel approach are shown in Fig. 2. Figure 2 also shows that due to physical properties of proton with no exit dose, it allows limitation of radiation doses to anteriorly placed OARs. In our study population, average doses receive by lens was $1.76 \%$ of prescribed dose, while cornea received $1.34 \%$ dose with $>/=96 \%$ dose coverage in cribriform plate region. It is important to achieve good coverage in cribriform plate region as it is one of the common sites of disease recurrence and its proximity to lens and cornea poses difficulty in radiation planning. To achieve better coverage in cribriform region and limit lens, optic apparatus radiation doses we used two posterior oblique fields for brain which in comparison to standard bilateral fields provided more than $98 \%$ of prescribed dose coverage without increasing lens dose (10).

Mean dose received by bilateral parotid glands was $4.63 \%$ for overall population and maximum dose received by oesophagus was $61.6 \%$ of prescribed dose; while average dose received by lungs, kidney and bowel was less than $0.1 \%$. Liver, heart and gonads did not receive any radiation dose. We did subgroup analysis by dividing these patients in paediatric (less than 14 years) and adult age group $(>/=14$ years), as all our patients less than 14 years received whole vertebra CSI. In adult patients, radiation dose to all OARs was less than $0.1 \%$ of prescribed dose as shown in Table 2 . For paediatric patients in whom whole vertebral CSI was planned, we modified standard contouring guidelines as in this group of patients with standard contouring guidelines, vital OARs such as midline mucosa, oesophagus, thyroid gland and bowel bag are partially in PTV volume (as shown in sagittal Fig. 2 IIA, and axial section Fig. 2 IIB). And this causes either significant dose delivery to the OARs up to $100 \%$ of prescribed dose or significant underdosing in the target volume. We mitigated this with our modified contouring method in paediatric patients receiving proton $\mathrm{CSI}$, and like discussed above. We instead of accepting under-dosing or sharp dose gradient in vertebral body region, accepted uniform low dose and verified vertebral body coverage 
while radiation plan robustness (3 $\mathrm{mm}$ translational shifts and $3.5 \%$ range uncertainties) was done for CTV including spinal canal and nerve roots. This novel approach, allowed better OAR sparing with no or minimal target underdosing, no dose gradient in vertebral body region.

Dosimetric benefit of proton therapy for CSI is translated in reduction radiation dose to OARs which in turn reduces acute toxicities and improves compliance to treatment. Acute toxicities in our cohort were remarkably less. In overall population most common acute toxicity was dermatitis, $50 \%$ patient had grade one dermatitis while remaining 50\% developed grade 2 dermatitis and no patient developed grade 3 or more dermatitis. All patients developed dermatitis in scalp region near boost volume and no patient developed grade 2 or more dermatitis in back region near spinal target volume.

Out of these 40 patients, only $8(20 \%)$ developed grade 1 mucositis while $2(5 \%)$ patients developed grade 2 mucositis and one (2.5\%) developed grade 3 mucositis. In subgroup analysis, only one patient aged more than 14 years developed grade 1 mucositis, no one developed grade 2 or more mucositis. In patients aged less than 14 years, $22 \%$ developed grade 1 mucositis while only two patients (6\%) developed grade 2 mucositis and one (3\%) developed grade 3 mucositis. Only patient who developed grade 3 mucositis was first paediatric patient receiving whole vertebral body CSI using PBT and contouring was done as per the standard SIOP guidelines (Fig. 2 IIA, B). In addition, patient was receiving concurrent weekly injection vincristine. After 5 fractions he developed grade 3 esophagitis, required treatment break, oral opioid analgesics and intravenous fluid administration with supportive care. For him plan adaption was done with partial underdosing od vertebral body in PRV region to limit OAR doses as per Tasson et al (17). After this we reviewed existing literature and mitigated this issue with modified approach as discussed above, and with this approach no paediatric patient developed grade 3 or more toxicities.

Haematological toxicities were noticed in $60 \%$ of all patients, $15 \%$ patients had grade 1 neutropenia, $7.5 \%$ had grade 2 neutropenia and only four (10\%) patients developed grade 3 neutropenia. Again grade 3 neutropenia was seen only in paediatric patients either receiving chemotherapy or re-irradiation. Only 3 patients developed grade 1 thrombocytopenia, all were receiving concurrent chemotherapy and of these 2 were from paediatric age group. Four patients had decrease in haemoglobin, 3 had grade 1 decrease while one had grade 2; none developed grade 3 anaemia. Gastrointestinal toxicities were noted in 10 patients. No patient in this cohort developed grade 2 or more weight loss during treatment and median weight loss was $4.8 \%$ of baseline weight, 3 patients lost weight more than $10 \%$ of their baseline, all were paediatric age group. No patient developed grade 2 or more anorexia, nausea vomiting during treatment, grade 1 anorexia was noted in $7(23.3 \%)$ patients while $6(20 \%)$ had grade 1 nausea vomiting.

Hospital admission was required in five patients, all were age less than 10 years, reason for hospital admission was either supportive care, symptomatic treatment or management of neutropenia or VP shunt management. Average hospital stay as we mentioned above was 2 days and this admission during treatment did not prolong overall treatment time for these patients. Three patients, 2 paediatric and one adult patient underwent plan adaptation during treatment, trigger for adaptation was daily CBCT in two 
patients while one had clinical indication. Reason for plan adaptation were either set up errors due to change in patient characteristics $(67 \%)$ or acute mucositis $(33 \%)$.

At median follow up of 12 months, of these 40 patients, $83.3 \%$ patients are alive and of them $92 \%$ were alive with no evidence of disease (Fig. 3A). We did subgroup analysis, as this cohort had different diagnosis which are strong determinant of their treatment outcomes. We divided patients according to diagnosis in three groups, group one included patients with medulloblastoma, group two included recurrent ependymoma patients receiving CSI as re-irradiation and others were included in third group.

In medulloblastoma group, 19 out of 20 patients are alive with overall survival of $95 \%$ at median follow up of 12 months. In recurrent ependymoma group OS was $71.4 \%$ while in third group OS was $76.9 \%$ (Fig. 3B). When we compared our toxicity and survival outcomes with available proton publications, our patients had comparable or slightly better toxicity profile and similar survival outcomes. Barney et al in audit of 50 adult CSI patients treated with proton therapy with similar median CSI dose at median follow up of 20 months, noticed median weight loss of $1.6 \%$, grade 1 nausea/vomiting in $46 \%$, grade 2 nausea in $20 \%$ and $10 \%$ patient had grade 2 or more anorexia, while four patients had grade 3 or more cytopenia (18). In comparison in our cohort of adult patients, median weight loss was marginally less $(1.6 \% \mathrm{Vs}$ $1.38 \%$ ), while none had nausea/vomiting, anorexia and cytopenia.

McGovern et al in their experience of 14 paediatric patients received CSI using PBT, documented grade 3 or more haematological toxicities (Neutropenia, thrombocytopenia and or anaemia) in 50\% of patients, while one developed sepsis during the treatment (19). In comparison, in our cohort only one (4.5\%) patient developed grade 3 neutropenia. Considering different histopathologies in both groups survival outcomes are not comparable.

In addition to this toxicity profile, we expect significant risk reduction in late toxicities and risk of second malignant neoplasm in both adult and paediatric patients treated with proton therapy as predicted by various models (20-22), especially in medulloblastoma group where long survivorship is reported (23). Superior toxicity profile and compliance to treatment in both adult as well as paediatric patients can be attributed to holistic care for each patient, combined team efforts of medical oncologist, dietician, physiotherapist and psychotherapist along with treating physician. We are following standard practice of regular weekly reviews, starting nutritional supplements at the start of treatment and low threshold for intervention. In addition pencil beam scanning technique with daily on board imaging and regular quality assurance CT scan imaging ensured accurate treatment delivery.

Limitations of this study includes a relatively short follow up, mixed age groups and histologies included. We would like to continue follow-up of these patients, document quality of life, late toxicity outcomes and survival outcomes for this cohort.

\section{Conclusion}


We report successful implementation of image guided proton CSI from India. Acute toxicity profile, compliance to treatment and early outcomes of modern proton therapy with pencil beam scanning technique and on board imaging for CSI in both adult and paediatric patients are encouraging. It is important to formulate separate contouring consensus guidelines for whole vertebral body irradiation in paediatric patients, to further extend dosimetric benefits of protons.

\section{Abbreviations}

\begin{tabular}{|ll|}
\hline Abbreviation & Full Form \\
\hline CSI & Cranio-spinal irradiation \\
\hline ATRT & Atypical Teratoid Rhabdoid Tumor \\
\hline PNET & Primitive Neuro-Ectodermal Tumor \\
\hline APL & Acute promyelocytic leukemia \\
\hline PTV & Planning Target Volume \\
\hline CTV & Clinical Target Volume \\
\hline D Max & Maximum dose \\
\hline D Mean & Mean dose \\
\hline Avg & Average \\
\hline
\end{tabular}

\section{Declarations}

Ethics approval and consent to participate: Institutional Ethics Committee - Bio Medical Research, Apollo Hospitals, Chennai, India has approved the study

Consent for publication: As per institutional protocols

Availability of data and materials: All data generated or analysed during this study are included in this published article [and its supplementary information files].

Competing interests: The authors declare that they have no known competing financial interests or personal relationships that could have appeared to influence the work reported in this paper.

Funding: None received

Authors' contributions: All authors have contributed towards the study and development of the manuscript.

Acknowledgements: The authors would like to acknowledge all the patients, their families and all members of the Neuro-Oncology and Paediatric Cancer Management Teams 


\section{References}

1. Paterson E, Farr RF. Cerebellar medulloblastoma: treatment by irradiation of the whole central nervous system. Acta Radiol. 1953 Apr;39(4):323-36.

2. Tsang DS, Murray L, Ramaswamy V, Zapotocky M, Tabori U, Bartels U, et al. Craniospinal irradiation as part of re-irradiation for children with recurrent intracranial ependymoma. Neuro-Oncol. 2019 Mar 18;21(4):547-57.

3. Farnia B, Allen PK, Brown PD, Khatua S, Levine NB, Li J, et al. Clinical outcomes and patterns of failure in pineoblastoma: a 30-year, single-institution retrospective review. World Neurosurg. 2014 Dec;82(6):1232-41.

4. Brandes AA, Pasetto LM, Monfardini S. The treatment of cranial germ cell tumours. Cancer Treat Rev. 2000 Aug;26(4):233-42.

5. Wong KK, Ragab O, Tran HN, Pham A, All S, Waxer J, et al. Acute toxicity of craniospinal irradiation with volumetric-modulated arc therapy in children with solid tumors. Pediatr Blood Cancer. 2018 Jul;65(7):e27050.

6. Johnstone PAS, McMullen KP, Buchsbaum JC, Douglas JG, Helft P. Pediatric CSI: are protons the only ethical approach? Int J Radiat Oncol Biol Phys. 2013 Oct 1;87(2):228-30.

7. Mahajan A. Proton Craniospinal Radiation Therapy: Rationale and Clinical Evidence. Int J Part Ther. 2014 Sep 8;1(2):399-407.

8. Seravalli E, Bosman M, Lassen-Ramshad Y, Vestergaard A, Oldenburger F, Visser J, et al. Dosimetric comparison of five different techniques for craniospinal irradiation across 15 European centers: analysis on behalf of the SIOP-E-BTG (radiotherapy working group). Acta Oncol Stockh Swed. 2018 Sep;57(9):1240-9.

9. Ajithkumar T, Horan G, Padovani L, Thorp N, Timmermann B, Alapetite C, et al. SIOPE - Brain tumor group consensus guideline on craniospinal target volume delineation for high-precision radiotherapy. Radiother Oncol J Eur Soc Ther Radiol Oncol. 2018 Aug;128(2):192-7.

10. Robustly optimized hybrid intensity-modulated proton therapy for craniospinal irradiation. 2021 Aug 29 [cited 2021 Aug 29]; Available from: https://www.cancerjournal.net/preprintarticle.asp? id $=324038$,type $=0$

11. Noufal MP, Sharma SD, Patro K, Arjunan M, Krishnan G, Tyagarajan R, et al. Impact of spot positional errors in robustly optimized intensity-modulated proton therapy plan of craniospinal irradiation. Radiol Phys Technol. 2021 Sep;14(3):271-8.

12. Chilukuri S, Panda PK, Jalali R. Proton Therapy in LMICs: Is the Need Justified? JCO Glob Oncol. 2022 Jan;8:e2100268.

13. Tonse R, Noufal MP, Deopujari CE, Jalali R. India's First Proton Beam Therapy Pediatric Patient. Neurol India. 2020 Feb;68(1):189-91.

14. Chilukuri S, Burela N, Uppuluri R, Indumathi D, Nangia S, Panda PK, et al. Preliminary Experience of Treating Children and Young Adults With Image-Guided Proton Beam Therapy in India. JCO Glob 
Oncol. 2020 Nov 1;(6):1736-45.

15. Pediatric proton therapy in 2015: Indications, applications and considerations [Internet]. [cited 2021 Aug 28]. Available from: https://www.appliedradiationoncology.com/articles/pediatric-protontherapy-in-2015-indications-applications-and-considerations

16. Lundkvist J, Ekman M, Ericsson SR, Jönsson B, Glimelius B. Proton therapy of cancer: potential clinical advantages and cost-effectiveness. Acta Oncol Stockh Swed. 2005;44(8):850-61.

17. Tasson A, Laack NN, Beltran C. Clinical Implementation of Robust Optimization for Craniospinal Irradiation. Cancers. 2018 Jan 3;10(1):E7.

18. Barney CL, Brown AP, Grosshans DR, McAleer MF, de Groot JF, Puduvalli V, et al. Technique, outcomes, and acute toxicities in adults treated with proton beam craniospinal irradiation. NeuroOncol. 2014 Jan;16(2):303-9.

19. McGovern SL, Okcu MF, Munsell MF, Kumbalasseriyil N, Grosshans D, McAleer MF, et al. Outcomes and acute toxicities of proton therapy for pediatric atypical teratoid/rhabdoid tumor of the CNS. Int $\mathrm{J}$ Radiat Oncol Biol Phys. 2014 Dec 1;90(5):1143-52.

20. Miralbell R, Lomax A, Cella L, Schneider U. Potential reduction of the incidence of radiation-induced second cancers by using proton beams in the treatment of pediatric tumors. Int J Radiat Oncol Biol Phys. 2002 Nov 1;54(3):824-9.

21. Taddei PJ, Mahajan A, Mirkovic D, Zhang R, Giebeler A, Kornguth D, et al. Predicted risks of second malignant neoplasm incidence and mortality due to secondary neutrons in a girl and boy receiving proton craniospinal irradiation. Phys Med Biol. 2010 Dec 7;55(23):7067-80.

22. Newhauser WD, Fontenot JD, Mahajan A, Kornguth D, Stovall M, Zheng Y, et al. The risk of developing a second cancer after receiving craniospinal proton irradiation. Phys Med Biol. 2009 Apr 21;54(8):2277-91.

23. Salloum R, Chen Y, Yasui Y, Packer R, Leisenring W, Wells E, et al. Late Morbidity and Mortality Among Medulloblastoma Survivors Diagnosed Across Three Decades: A Report From the Childhood Cancer Survivor Study. J Clin Oncol. 2019 Mar 20;37(9):731-40.

\section{Figures}

\section{Figure 1}

Age distribution (a), diagnosis and molecular subgrouping (b).

\section{Figure 2}

Cranio-spinal dose distribution 
IA - CSI in young adult, sagittal view

IB - CSI in young adult, axial view

IIA - CSI in paediatric patient, standard SIOP guidelines, sagittal view

IIB - CSI in paediatric patient, standard SIOP guidelines, axial view

IIIA - CSI in paediatric patient, modified approach, sagittal view

IIIB - CSI in paediatric patient, modified approach, axial view

Colours - Red Isodose showing $98 \%$ of prescribed dose

Orange Isodose showing $95 \%$ of prescribed dose

Yellow Isodose showing $80 \%$ of prescribed dose

Dark Green Isodose showing $70 \%$ of prescribed dose

Light Blue Isodose showing $50 \%$ of prescribed dose

Light Green Isodose showing $30 \%$ of prescribed dose

Dark blue Isodose showing $10 \%$ of prescribed dose 

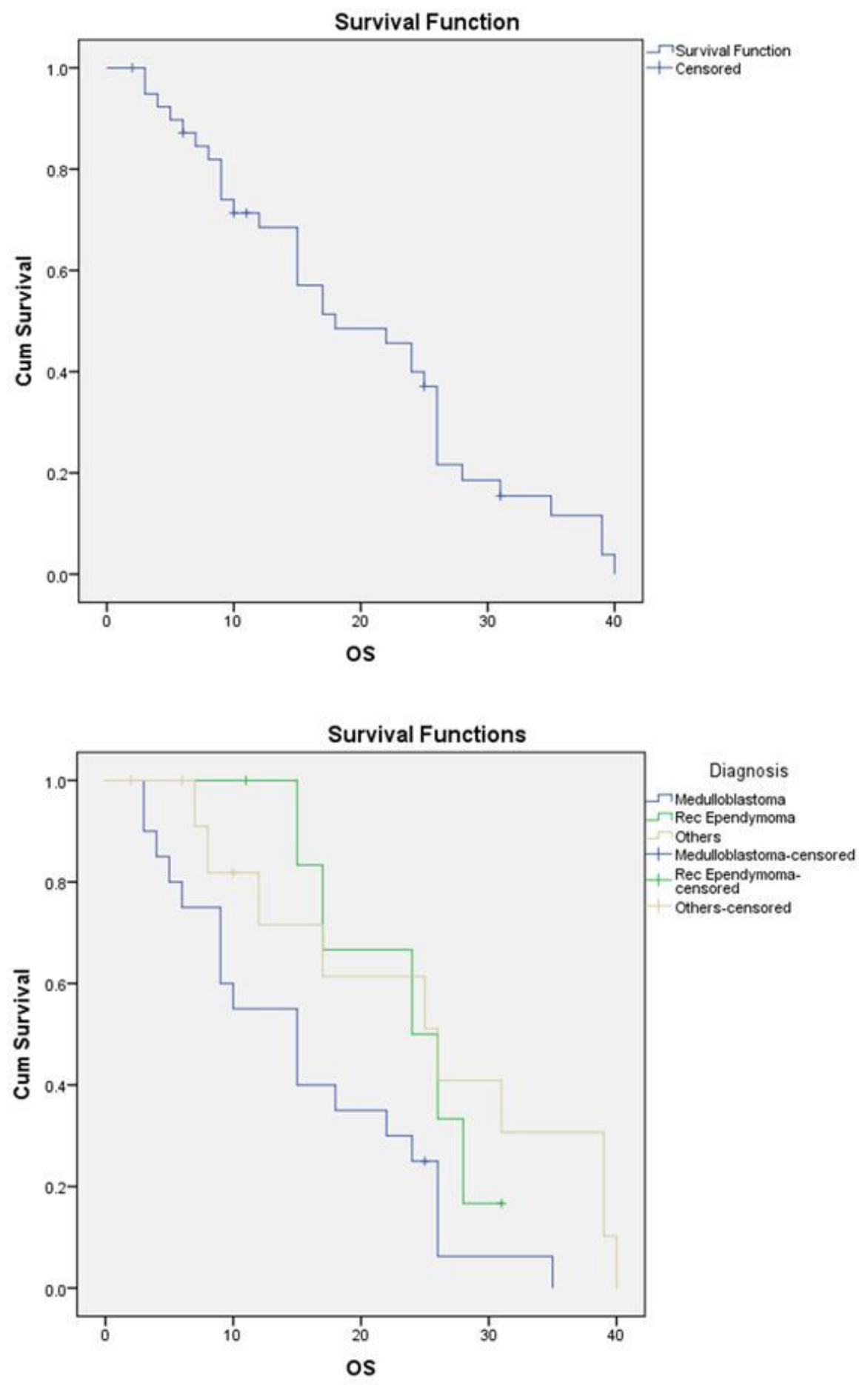

Figure 3

Survival curves

$3 A$ - For overall cohort

B - For Histological subgroups 
Page 17/17 